\title{
45
}

\section{Multiplexing periodic sources in a tree network of ATM multiplexers*}

\author{
J.M. Barceló, J. García
}

Polytechnic University of Catalonia

Computer Architecture Department, c/ Gran Capitan, Modulo C6E105, Barcelona E-08071, Spain

tel $:+3434016798$, fax $:+3434017055$,

e-mail: joseb@ac.upc.es, jorge@ac.upc.es

\begin{abstract}
We obtain the queue length distribution in a tree of discrete time queues with constant service time whose input is periodic traffic. In the context of ATM the study could be applied to CBR sources. The tree consists of Mstages. To solve this system, we first solve a 2 -stage tree network. Given this configuration, a more complex tree network can be easily solved making use of the properties of the discrete time queues with identical service times. We also give closed formulas for the average waiting time and average number of cells in any queue of the tree network.
\end{abstract}

ATM, tree topology, periodic sources

\section{Keywords}

\section{INTRODUCTION.}

In this paper we derive closed-form formulas for the queue length distributions in a discrete-time $\mathrm{M}$-stage tree queueing network loaded with periodic traffic sources. In this type of network, the queues can be grouped in $M$ groups or stages. Every queue of a stage is fed by all the exit traffic of any given number (which could be 0 ) of queues from the previous stage as well as by certain number (which could be 0 ) of external sources of traffic. All the entrance traffic in the network is routed to the root queue, which occupies the first stage (see in Figure 5 an example for the case $M=3$ ). We consider the discrete-time case in which all the queues have a single server with constant service time. As is shown in Morrison (1978) only two configurations are relevant to solve this system: the case $M=1$, which corresponds to a single server queue with a constant service time and the case $M=2$. The solution of the case $M>2$ can be found solving systems with $M=1$ and $M=2$.

We are interested in tree networks of discrete time queues with constant ser- 
vice time whose input is periodic traffic (e.g. CBR sources or periodic sources with back-to-back cells). For this kind of traffic, we compute the probability distribution function of the virtual waiting time in any queue of the tree.

In Modiano et al (1996) a solution to the average queuing delay in a tree network of discrete time queues with constant service time where the arrival process is Poisson is presented. Their solution is based on an equivalent network where priority is given to customers in transit. From this priority model it is easy to derive the average waiting time and average number of clients in the system in any queue of the tree. We will make use of this result to give a closed formula for the average waiting time when the input traffic is periodic. We think that although this kind of topology is limited, its solution can lead to give us more insight to more general queue systems such as queues in tandem with cross traffic.

The paper is organized as follows: in section 2, we compute the CPDF (Complementary Probability Distribution Function) of the virtual waiting time in this kind of topologies with periodic traffic. We first calculate the case of two multiplexing stages, to later extend the solution to M-stage networks. In section 3, we apply the equivalent priority model proposed in Modiano et al (1996) to obtain the average waiting time and average number of cells in the system in any queue of the tree network. Finally we give some results in section 4 .

\section{MULTIPLEXING PERIODIC SOURCES IN AN M-STAGE TREE QUEUEING NETWORK.}

\subsection{Two-stage tree network.}

In this section we compute the CPDF for the multiplexing of periodic sources in two-stage tree networks as a first step to later extend it to M-stage networks. Each source is periodic and emits a constant number, $b$, of back-to-back cells remaining silent during the rest of the period, T-b slots. The time at which the burst becomes active is uniformly and independently distributed within the period, see Figure 1. By choosing $b=1$, we have CBR sources. We consider the pooling of $K$ buffers into a single queue, the root queue, which receives also $N_{r}$ sources as input (called exogenous traffic). Each one of the $i(\mathrm{i}=1 \ldots \mathrm{K}$ ) buffers multiplexes $N_{i}$ sources. We consider every source in the network to have identical characteristics, burst $b$ and period $T$, and every queue to have a constant service time of one ATM slot. In fact the hypothesis that every source has the same characteristics can be relaxed to the hypothesis that the external sources which feed a given queue have the same characteristics and all the sources which enter the network are of equal period. We assume stability 
at each queue of the tree considering that $N_{i} b<T$ for all $i=1, \ldots K$ and $\left(\sum_{i=1}^{K} N_{i}+N_{r}\right) \cdot b<T$.

We are interested in the distribution of the queue length at the root queue. The distribution of the Virtual Waiting time of a single queue system fed by periodic sources can be computed, for instance, by means of the Benes bound, see Roberts and Virtamo (1991) for the multiplexing of periodic sources emitting one cell and see García et al (1995) for the multiplexing of periodic sources emitting bursts of $b$ cells. For simplicity's sake we substitute the burst arrivals of the sources which feed the second stage queues by batch arrivals of size $b$. This does not suppose any change in the results for the root queue, see Figure 2. Therefore, when we calculate the queue length distribution in the root queue we assume that each source which enters the queues of the second stage generates the following pattern: a batch of $b$ cells followed by a silence of $T-1$ slots, see Figure 1. The arrival epoch of the batches also is independently and uniformly distributed within the period $T$.

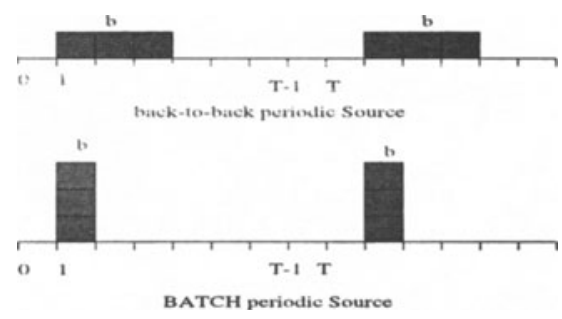

Figure 1 Back-toback and Batch periodic sources.

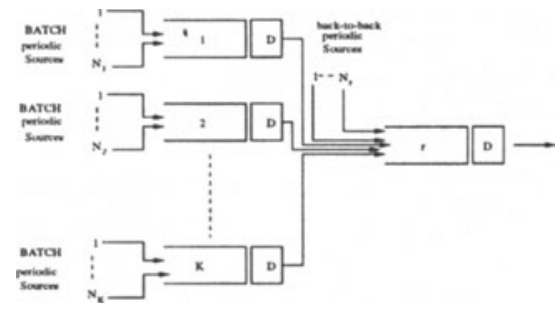

Figure 2 Twostage tree network.

We define $N^{r}(t, 0)$ as the number of cell arrivals to the root queue $r$ in the interval [-t,0), and $L_{-t}^{r}$ as its queue length at time -t. We can express $P\left\{L_{0}^{r}>x\right\}$ as:

$$
P\left\{L_{0}^{r}>x\right\}=\sum_{t=1}^{T} P\left\{N^{r}(t, 0)=t+x \text { and } N^{r}(u, 0)<u+x, t<u \leq T\right\}
$$

Note that events that occur in the queues of the second stage at any time will influence the root queue one slot later. We define the following functions:

- $B^{r}\left(t_{1}, t_{2}\right)$ is the number of exogenous sources that become active in an interval $\left[-t_{1},-t_{2}\right)$ in the root queue.

- $B^{i}\left(t_{1}, t_{2}\right)$ is the number of batch arrivals to the $i$-th queue in an interval $\left[-t_{1},-t_{2}\right)$, with $i=1, \cdots, K$.

- $G\left(t, t+x, n_{1}, \ldots, n_{K}, n_{r}\right)=P\left\{N^{r}(t, 0)=t+x \quad \mid \quad N^{r}(u, 0)<u+x, t<u \leq\right.$ $\left.T, B^{1}(t+1,1)=n_{1} \ldots, B^{K}(t+1,1)=n_{K}, B^{r}(t, 0)=n_{r}\right\}$

- $L\left(t, n_{1}, \ldots, n_{K}, n_{r}\right)=P\left\{N^{r}(u, 0)<u+x, t<u \leq T \quad \mid \quad B^{1}(t+1,1)=n_{1} \ldots, B^{K}(t+\right.$ $\left.1,1)=n_{K}, B^{r}(t, 0)=n_{r}\right\}$ 
- $B\left(t, n_{1}, \ldots, n_{K}\right)=P\left\{B^{1}(t+1,1)=n_{1}, \ldots, B^{K}(t+1,1)=n_{K}\right\}$.

Equation (1) can be transformed into:

$$
\begin{aligned}
P\left\{L_{0}^{r}>x\right\}=\sum_{t=1}^{T} \sum_{n_{1}=0}^{N_{1}} . . \sum_{n_{K}=0}^{N_{K}} \sum_{n_{r}=0}^{N_{r}} G\left(t, t+x, n_{1}, \ldots, n_{K}, n_{r}\right) . \\
\cdot L\left(t, n_{1}, \ldots, n_{K}, n_{r}\right) \cdot B\left(t, n_{1}, \ldots, n_{K}\right) \cdot P\left\{B^{r}(t, 0)=n_{r}\right\}
\end{aligned}
$$

(a) Term $G\left(t, t+x, n_{1}, \ldots, n_{K}, n_{r}\right)$ :

In order to obtain this term we should bear in mind that the event $\left\{N^{r}(u, 0)<\right.$ $u+x, t<u \leq T\}$ implies that the $K$ queues are empty at time $-(\mathrm{t}+1)$. Therefore the contribution which each queue of the second stage makes to the root queue during the interval $[-t, 0)$ can only have as its origin the cells which arrive at the said queue during the interval $[-(t+1),-1)$. We introduce now the following notation:

- $N^{r i}(t, 0)$ is the number of cells that arrive at the root queue $r$ from the $i$-th queue in the interval $[-t, 0)$.

- $R^{r i}\left(t, k_{i}, n_{i}\right)$ is the probability $P\left\{N^{r i}(t, 0)=k_{i} \quad \mid\right.$ queue $i$ is empty at $-(t+1)$, $\left.B^{i}(t+1,1)=n_{i}\right\}$.

- $N^{r r}(t, 0)$ is the number of cells that arrive at queue $r$ from the exogenous sources.

- $R^{r r}\left(t, k_{r}, n_{r}\right)$ is $P\left\{N^{r r}(t, 0)=k_{r} \mid\right.$ queue $r$ is empty at $\left.-t, B^{r}(t, 0)=n_{r}\right\}$.

Probability $G\left(t, t+x, n_{1}, \ldots, n_{K}, n_{r}\right)$ will be the convolution of $R^{r i}(i=$ $1, \ldots K)$ and $R^{r r}$ :

$$
G\left(t, t+x, n_{1}, \ldots, n_{K}, n_{r}\right)=\sum_{\sum_{j=1}^{K} k_{i}+k_{r}=t+x} \prod_{i=1}^{K} R^{r i}\left(t, k_{i}, n_{i}\right) \cdot R^{r r}\left(t, k_{r}, n_{r}\right)
$$

(b) Terms $R^{r r}\left(t, k_{r}, n_{r}\right)$ and $R^{r i}\left(t, k_{i}, n_{i}\right)$ :

The term $R^{r r}\left(t, k_{r}, n_{r}\right)$ can be obtained from a partial result obtained in García et al (1995). In that system, three regions were considered: $t<b$, $b \leq t<T-b$ and $T-b \leq t<T$. For a proof of this result we refer to García et al (1995). Term $R^{r r}\left(t, k_{r}, n_{r}\right)$ is then expressed as:

$$
\left\{\begin{array}{l}
\frac{1}{t^{n_{r}}} q_{t}^{\left(n_{r}\right)}\left(k_{r}\right) \quad \text { if } t<b, \quad n_{r} \leq N_{r} \\
\sum_{j=0}^{n_{r}}\left(\begin{array}{c}
n_{r} \\
j
\end{array}\right) \frac{(t-b+1)^{j}}{t^{n_{r}}} q_{(b-1)}^{\left(n_{r}-j\right)}\left(k_{r}-b j\right) \text { if } \quad b \leq t<T-b, n_{r} \leq N_{r} \\
\sum_{j=0}^{N}\left(\begin{array}{c}
N_{r} \\
j
\end{array}\right) \frac{(t-b+1)^{j}}{t^{N_{r}}} q_{(b-1)}^{\left(N_{r}-j\right)}\left(k_{r}-b j\right) \quad \text { if } \quad T-b \leq t<T, n_{r}=N_{r}
\end{array}\right.
$$


Where $q_{a}(y)$ is the discrete-time unitary pulse in the interval $[1, \mathrm{a}]$ and $q_{a}^{(k)}(y)$ is its $k$-th discrete-time convolution. A simple expression for $q_{a}^{(k)}(y)$ is derived in García et al (1995) (see also Feller):

$q_{a}^{(k)}(y)=\sum_{s=0}^{\left\lfloor\frac{y-k}{a}\right\rfloor}(-1)^{s}\left(\begin{array}{c}k \\ s\end{array}\right)\left(\begin{array}{c}y-s \cdot a-1 \\ k-1\end{array}\right)$ if $k>0$ for $y=k, \ldots, k a(5)$

And $q_{a}^{(k)}(y)=0$ for other values of $y$. For $k=0$ we define $q_{a}^{(0)}(y)=\delta(y)$, where $\delta(y)$ is the discrete-time impulse function.

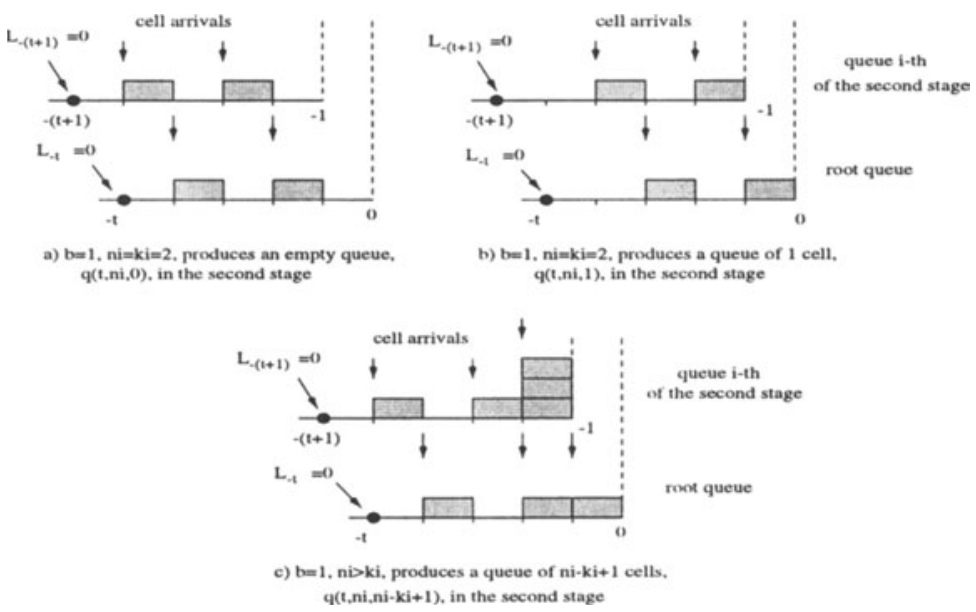

Figure 3 Cases to calculate the term $R^{r i}\left(t, k_{i}, n_{i}\right)$, (with $\left.\mathrm{b}=1\right)$.

To calculate the term $R^{r i}\left(t, k_{i}, n_{i}\right)$, we must take into account the fact that the temporal axis in the queues of the second stage is shifted one time slot (the arrivals which are relevant to the root queue in the time instant $t=0$ are produced in the queues of the second stage during the interval $[-(T+1),-1)$ ). Since each queue begins empty, we can substitute queue $i$ for a queue of period $t$ in the interval $[-(\mathrm{t}+1),-1)$, to which there arrive $n_{i}$ batches of size $b$ uniformly and independently distributed. It follows that, if $n_{i} b$ cells arrive at queue $i$ and $k_{i}$ cells are emitted, at time instant $t=-1$ there will be either a queue of length $n_{i} b-k_{i}+1$ (in the case $n_{i} b>k_{i}$, see Figure 3.c) or a queue 
of length 0 or 1 (in the case $n_{i} b=k_{i}$, see Figures 3.a,b). In consequence we will have the following cases:

$$
R^{r i}\left(t, k_{i}, n_{i}\right)=\left\{\begin{array}{llccc}
1 & \text { if } & k_{i}=0 & \text { and } & n_{i}=0 \\
0 & \text { if } & k_{i}=0 & \text { and } & n_{i}>0 \\
0 & \text { if } & k_{i}>0 & \text { and } & n_{i} \cdot b<k_{i} \\
q_{b}\left(t, n_{i}, 0\right)+q_{b}\left(t, n_{i}, 1\right) & \text { if } & k_{i}>0 & \text { and } & n_{i} \cdot b=k_{i} \\
q_{b}\left(t, n_{i}, n_{i} b-k_{i}+1\right) & \text { if } & k_{i}>0 & \text { and } & n_{i} \cdot b>k_{i}
\end{array}\right.
$$

Where $q_{b}(T, N, x)$ is the probability that the queue length of an $N D / D / 1$ with period $T$ and $N$ batch arrivals of length b is $x$. As it is easily demonstrated, the expression $q_{b}(T, N, x)$ is equal to $Q_{b}(T, N, x-1)-Q_{b}(T, N, x)$, where for $T+x>N b$ :

$Q_{b}(T, N, x)=\sum_{i=\left\lceil\frac{x}{b}\right\rceil}^{N}\left(\begin{array}{c}N \\ i\end{array}\right)\left(\frac{i b-x}{T}\right)^{i}\left(1-\frac{i b-x}{T}\right)^{N-i}\left(1-\frac{(N-i) b}{T-i b+x}\right)$

(c) $\operatorname{Term} L\left(t, n_{1}, \ldots, n_{K}, n_{r}\right)$ :

To calculate $L\left(t, n_{1}, \ldots, n_{K}, n_{r}\right)=P\left\{N^{r}(u, 0)<u+x, t<u \leq T \quad \mid \quad B^{1}(t+\right.$ $\left.1,1)=n_{1}, \ldots, B^{K}(t+1,1)=n_{K}, B^{r}(t, 0)=n_{r}\right\}$ we use similar arguments as in Roberts and Virtamo (1991) or García et al (1995). The event $P\left\{N^{r}(u, 0)<\right.$ $\left.u+x, t<u \leq T \quad \mid \quad B^{1}(t+1,1)=n_{1}, \ldots, B^{K}(t+1,1)=n_{K}, B^{r}(t, 0)=n_{r}\right\}$ corresponds to the situation in which the root queue of an auxiliary network of queues loaded with periodic arrivals of period $T-t$ in the interval $[-(\mathrm{T}-\mathrm{t}), 0)$ is empty at time 0 .

During a period, the root queue of the said auxiliary network receives cells with different origins, see Figure 4.b:

- cells which come from the exits of the second stage queues,

- cells which come from bursts generated by the external sources which directly feed the root queue and that began during the actual period, and

- cells which come from bursts generated by the external sources which directly feed the root queue and that began in a previous period but that in part contribute to the actual period.

The cells which enter the root queue from the second stage queues in the auxiliary system are those which arrived at the second stage queues in $N_{i}-n_{i}$ batches of $b$ cells uniformly distributed in the interval [-(T-t+1),-1). At this point it is important to note that for the auxiliary root queue to be empty in the time instant $t=0$ it is necessary for the second stage queues to be empty too. This means that all cells which arrive at the second stage queues between $[-(T-t+1),-1)$ must be served beforehand and routed to the root queue at time instant $t=0$. 


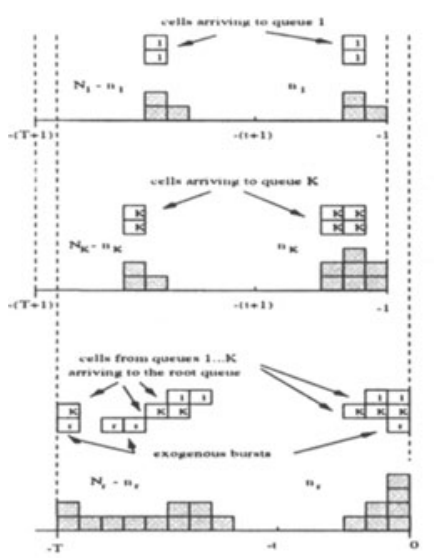

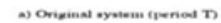

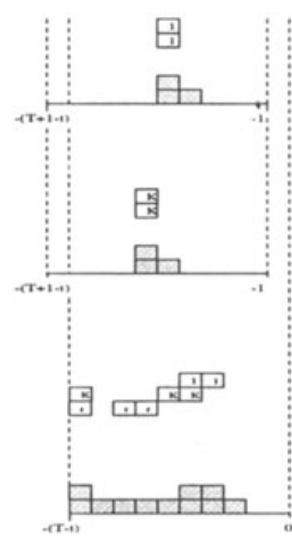

b) Aunatiany arotem (period T-t)

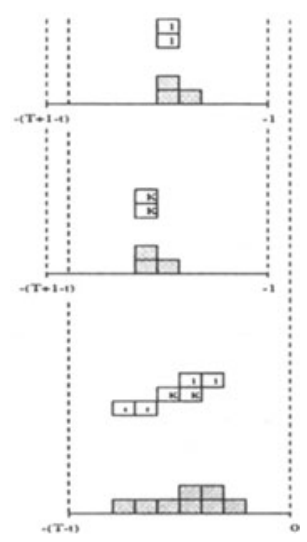

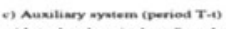

Figure 4 Equivalent system to calculate term $L\left(t, n_{1}, \ldots, n_{K}, n_{r}\right)$. a) Original system. b) Auxiliary system, where bursts from the auxiliary second stage queues and exogenous bursts plus cells belonging to bursts that began in the previous period arrive at the auxiliary root queue. c) Auxiliary system, where only bursts from the auxiliary second stage queues and exogenous bursts arrive at the auxiliary root queue

Arrivals which proceed directly from external sources and which feed the root queue in the auxiliary system consist of $N_{r}-n_{r}$ bursts of $b$ cells uniformly distributed in the interval $[-(\mathrm{T}-\mathrm{t}), 0)$.

Lastly, in the root queue of the auxiliary system we have an entrance in batch at the beginning of interval $[-(\mathrm{T}-\mathrm{t}), 0)$ with which we model the contribution of the bursts which (in the original system) began to be emitted in the previous period. This batch consists of $\sum_{i=1}^{K} n_{i} b+n_{r} b-t-x$ cells.

Since the original root systems is stable, $\sum N_{i} b+N_{r} b<T$, the auxiliary system will be so too: $\sum_{i=1}^{K}\left(N_{i}-n_{i}\right) b+\left(N_{r}-n_{r}\right) b+\sum_{i=1}^{K} n_{i} b+n_{r} b-t-x<$ $T-t$. Therefore the batch arrival at the beginning of the interval $[-(\mathrm{T}-\mathrm{t}), 0)$ will be served before the end of the interval, and will not contribute to the length of the queue in the instant $t=0$. We have then a situation in which the only arrivals which have to be counted in order to obtain an empty queue in the auxiliary root queue at time 0 are those which proceed from the exit of the $N_{i}-n_{i}$ batches and the $N_{r}-n_{r}$ exogenous sources (see Figure 4.c). Therefore:

$$
L\left(t, n_{1}, \ldots, n_{K}, n_{r}\right)=1-\frac{\left(\sum_{i=1}^{K}\left(N_{i}-n_{i}\right) \cdot b+\left(N_{r}-n_{r}\right) \cdot b\right)}{T-t}
$$


(d) Terms $P\left\{B^{r}(t, 0)=n_{r}\right\}$ and $B\left(t, n_{1}, \ldots, n_{K}\right)$ : $P\left\{B^{r}(t, 0)=n_{r}\right\}$ has a binomial distribution.

$P\left\{B^{r}(t, 0)=n_{r}\right\}=\left(\begin{array}{c}N_{r} \\ n_{r}\end{array}\right)\left(\frac{t}{T}\right)^{n_{r}} \cdot\left(1-\frac{t}{T}\right)^{N_{r}-n_{r}}$

Since each queue is independent and the batches are uniformly distributed, the term $B\left(t, n_{1}, \ldots, n_{K}\right)$ can be calculated as:

$B\left(t, n_{1}, \ldots, n_{K}\right)=\prod_{i=1}^{K} P\left\{B^{i}(t+1,1)=n_{i}\right\}=\prod_{i=1}^{K}\left(\begin{array}{c}N_{i} \\ n_{i}\end{array}\right)\left(\frac{t}{T}\right)^{n_{i}} \cdot\left(1-\frac{t}{T}\right)^{N_{i}-n_{i}}($

\subsection{M-stage tree network.}

Once the two stage system is solved, we can generalize the results for an $M$-stage tree network. Multiplexing stage $i$ is pooled by $K_{i-1}$ queues and $N_{p q}$ exogenous sources (with $q=1 \ldots K$ ). We can make use of a property of the rooted tree networks with discrete-time single server queues presented by Morrison (1978). Morrison showed that in such networks, the rooted tree network can be reduced to a two-stage network with a prescribed input. Specifically, to calculate the queue length distribution at the root queue, we can assume that the external sources that enter the queues which hang on each branch of a second stage queue, enter the second stage queue directly.

Using this argument, and the fact that each queue of the network is a root queue of a corresponding sub-tree, the analysis of the network with M-stages can be done from the analysis of equivalent systems with $M=1$ and $M=2$.

For example, consider a three multiplexing stage network, see Figure 5. Each queue is fed by exogenous sources. In order to obtain the queue length distribution at queue $r$ (the root queue), we can substitute this system for an equivalent two-stage network (Figure 6). In this equivalent system, only the sources which enter the root queue directly are back-to-back periodic sources. The rest behave as Batch periodic sources.

\section{AVERAGE DELAYS AND AVERAGE NUMBER IN THE QUEUE IN THE TREE NETWORK.}

To give close-form formulas for the average queueing delay and average waiting time in any queue of the tree network, we will make use of an equivalent network based on priorities proposed by Modiano et al (1996). In this proposal, they first consider a simple case with two queues in tandem and Poisson traffic as input. High-priority is given to customers in transit from one queue to the other, while low-priority is given to the exogenous traffic that enters directly 


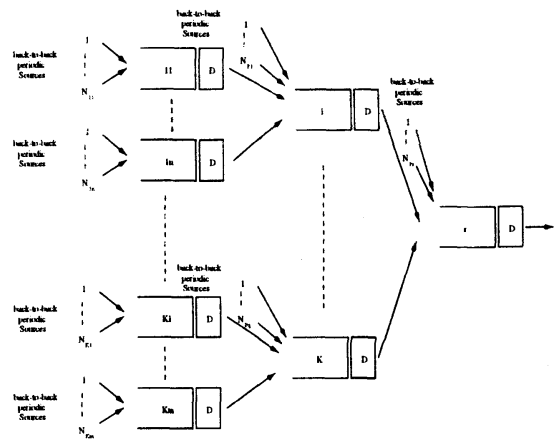

Figure 5 Three stage network.

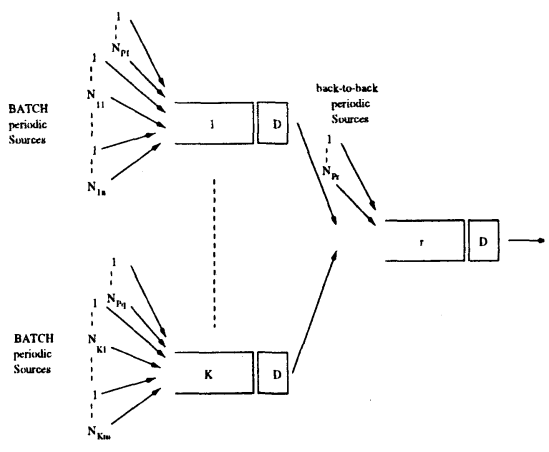

Figure 6 Equivalent network.

the first queue. If $\bar{Q}$ is the average overall queue size (low and high priority traffic), $\overline{Q_{1}}$ is the average queue size at the high priority queue and $\overline{Q_{2}}$ is the average queue size at the low priority queue, then it holds that $\overline{Q_{2}}=\bar{Q}-\overline{Q_{1}}$.

Solving this system, an extension to larger systems can be easily derived. If we consider a two stage system in which the second stage consists of $K$ queues whose output traffic is routed to the first stage as in Figure 2, the average number of customers in the root queue will be $\overline{Q_{r}}=\bar{Q}-\sum_{i \in K} \overline{Q_{i}}$.

Where $\overline{Q_{i}}$ is the average number of customers in queue $i=1, \ldots, K$ including queue room and service room and $\bar{Q}$ is the average number of customers in the total system except for those being serviced in the root queue.

We will apply this formula to calculate average delays in any queue system of the tree whose input is homogeneous periodic traffic of period $T$ and burst $b$. Dron et al (1991) show that the average waiting time in the queue, $\bar{W}$, of a queue system multiplexing $N$ CBR periodic sources of period $T_{c b r}$ with load $\rho=\frac{N}{T_{c b r}}$ is the following:

$\bar{W}=\frac{(N-1) !}{2} \sum_{k=1}^{(N-1)} \frac{1}{T_{c b r}^{k}} \frac{1}{(N-1-k) !}$

The number of cells in the queue $\bar{Q}$ can be calculated using Little's formula: in periodic systems this can be interpreted as following, see Humblet et al (1993):

$$
\begin{gathered}
P\{W=x\}= \\
P\{\text { a departure leaves } x-1 \text { cells in the queue }\}= \\
\frac{P\{Q=x\}}{P\{Q>0\}}=\frac{T_{c b r}}{N} P\{Q=x\} \text { with } x>0
\end{gathered}
$$


Therefore applying this relation and taking into account that the service time is the time unit:

$\bar{Q}=\rho \cdot \bar{W}=\frac{N !}{2 T_{c b r}} \sum_{k=1}^{(N-1)} \frac{1}{T_{c b r}^{k} \cdot(N-1-k) !}$

In the general case of multiplexing back-to-back periodic sources with burst size $b$ and period $T=b T_{c b r}$, the average waiting time in the queue can be expressed as $b$ times the average waiting time in the queue for an $N D / D / 1$ system with $N$ CBR sources of period $T_{c b r}$. Thus:

$$
\bar{Q}=b \overline{Q_{c b r}}=\frac{N ! b}{2 T_{c b r}} \sum_{k=1}^{(N-1)} \frac{1}{T_{c b r}^{k}} \frac{1}{(N-1-k) !}=\frac{N ! b^{2}}{2 T} \sum_{k=1}^{(N-1)}\left(\frac{b}{T}\right)^{k} \frac{1}{(N-1-k) !}
$$

We consider the same tree topology as in the former section. The second stage has $K$ queues fed each one by $N_{i}$ periodic sources of period $T=b \cdot T_{c b r}$ being $i=1, \ldots, K$ and $b$ the burst size. Let $\rho_{i}$ be the load at each queue $\left(\rho_{i}=\frac{N_{i} b}{T}\right)$. The output of each of these queue systems is multiplexed with $N_{r}$ exogenous periodic sources with load $\rho_{r}=\frac{N_{r} b}{T}$, see Figure 2. The total load in the root queue is $\rho=\sum_{i} \rho_{i}+\rho_{r}$. We define $N$ as the total number of sources in the system: $N=\sum_{i} N_{i}+N_{r}$. Therefore, we can express the average number of cells in the root queue as:

$\overline{Q_{r}}=\frac{N ! b^{2}}{2 T} \sum_{k=1}^{(N-1)} \frac{b^{k}}{T^{k}(N-1-k) !}-\sum_{i=1}^{K} \frac{N_{i} ! b^{2}}{2 T} \sum_{k=1}^{\left(N_{i}-1\right)} \frac{b^{k}}{T^{k}\left(N_{i}-1-k\right) !}$

The average waiting time in the queue is given by Little's formula $\overline{W_{r}}=$ $\frac{T}{N b} \overline{Q_{r}}$ and the average waiting time and average number of cells in the system can be expressed as: $\overline{W_{r_{s}}}=\overline{W_{r}}+1$ and $\overline{Q_{r_{s}}}=\overline{Q_{r}}+\rho$.

\section{RESULTS.}

In order to see how the number of second-stage queues influences the root queue, we have studied various configurations with different loads. In Figure 7 we have drawn the required buffer length in a root queue so that the probability of cell losses is below $10^{-10}$ as a function of $b$ when the second-stage has two queues. We call the number of sources which enter the second-stage queues $N_{1}$ and $N_{2}$ respectively. As can be seen in Figure 7 , the worst situation occurs with balanced loads (e.g. $N_{1}=N_{2}=6$ over a period $T=15 b$ ).

Finally in Figure 8, we show the queue length distribution when the second stage has $K=2,3,4,12$ queues. The curves are calculated with a total load 

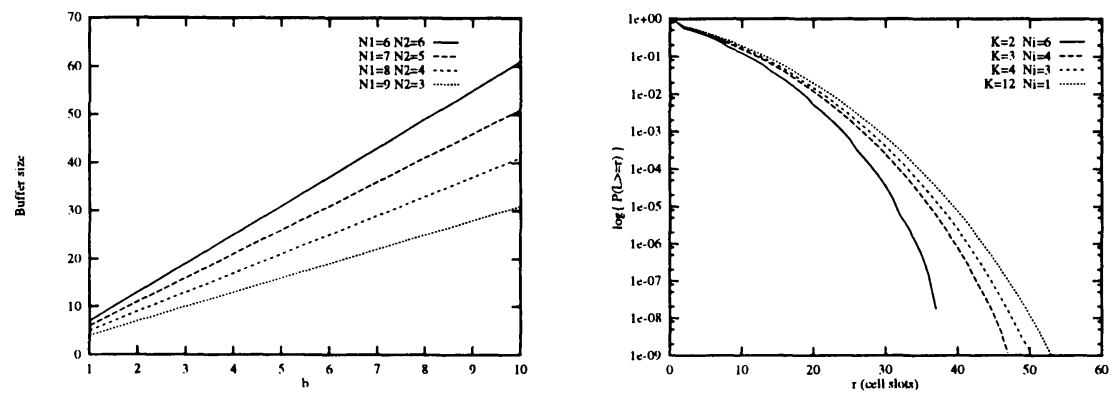

Figure 7 Buffer size in the root queue Figure 8 Queue length distribution for versus $b$. Two stages. $2,3,4,12$ queues in the second stage.

\begin{tabular}{ccccccc} 
& \multicolumn{3}{c}{ two stages, $\mathrm{K}=2$} & \multicolumn{3}{c}{ two stages, $\mathrm{K}=4$} \\
\hline$\rho$ & $T_{c b r}=100$ & $T_{c b r}=1000$ & $M / D / 1$ & $T_{c b r}=100$ & $T_{c b r}=1000$ & $M / D / 1$ \\
\hline 0.90 & 2.365 & 3.695 & 4.090 & 2.612 & 3.957 & 4.354 \\
\hline 0.80 & 1.286 & 1.610 & 1.666 & 1.482 & 1.817 & 1.875 \\
\hline
\end{tabular}

Table 1 Average waiting time in the queue for CBR sources and Poisson.

of $\rho=0.8$ and $b=6$. The period of the sources is $T=90$ and the load is balanced among the $K$ queues $\left(N_{k}=\frac{12}{K}\right.$ sources in each queue). The worst situation is when each queue is fed by only one source $(K=12)$. In that case, the same results would be obtained as if 12 sources were multiplexed in one stage.

In table 1 we compare the average waiting time in the queue, $\bar{W}$, for a root queue that is pooled by $K=2$ and $K=4$ buffers. In this case the load is balanced among the buffers. The average waiting time in the root queue is compared for the different periods and for the case in which Poisson traffic of load equal to the periodic traffic enters at each queue. We can observe what it is known: Poisson is pessimistic for high loads as in the single queue system. For $K=2$ the average delay decreases slightly respect to the case of $K=4$, but not too much. We also observe that if the number of buffers are increased it looks more like a single queue system.

\section{CONCLUSIONS.}

We have obtained the probability distribution function of the virtual waiting time in the root queue of a tree network of discrete time queues with constant service time whose input is periodic traffic. Using the Benes approach it is possible to compute the Virtual Waiting time CPDF in any queue of the tree network. For that purpose, we first solve a tree of two stages. The second stage consists of $K$ queues fed by periodic sources. The output of each of these queues is routed to the second stage and is multiplexed with exogenous 
periodic traffic. We assume that each queue either in the first stage or the second is stable.

Once the two-stage system is solved, we can make use of a property of the rooted tree networks with discrete-time single server queues presented by Morrison (1978) in to solve the M-stage queueing network. In such networks the external sources that feed queues that hang on a branch of a second stage queue, can be connected directly to the corresponding second stage queue root. In this way, we have an equivalent system of two stages.

To compute the average waiting time and the average number in the queue, we apply a model, presented in Modiano et al for Poisson traffic, based on an equivalent network where high priority is given to traffic in transit and low priority is given to exogenous traffic. We apply this method considering now that the input traffic is periodic and give closed formulas for the former average parameters.

\section{REFERENCES}

[1] ITU-T Recommendation I-371 (1995) "Traffic Control and Congestion Control in B-ISDN", Draft issue, Geneva, 1995.

[2] ATM Forum (1996) "ATM Forum Traffic Management Working Specification", Version 4.0, 1996

[3] Dron, L., Ramamurthy, G. and Sengupta, B. (1991) "Delay analysis of continuous Bit Rate Traffic over an ATM network", IEEE JSAC, Vol. 9, No 3, April 1991

[4] Feller, W. "An Introduction to Probability theory and its Applications", Vol 2, Ed. John Willey.

[5] García Vidal, J., Barceló, J.M. and Casals, O. (1995) "An Exact Model for the Multiplexing of Worst Case Traffic Sources", IFIP Conference on Performance on Computer Networks, PCN'95, Instambul, Turkey, October 1995.

[6] Humblet, P., Bhargava, A., and Hluichyi, M. (1993) "Ballot Theorem applied to the Transient Analysis of $n D / D / 1$ queues", IEEE/ACM Transactions on Networking, Vol 1, No 1, February 1993.

[7] Modiano, E., Wieselthier, J.E. and Ephemerides, A. (1996) "A simple analysis of average queueing delay in tree networks", IEEE Transactions on Information Theory, Vol. 42, No 2, March 1996

[8] Morrison, J.A. (1978) "A Combinatorial Lemma and its Application to Concentrating Trees of Discrete-Time Queues", The Bell System Technical Journal, Vol. 57, No 5, May-June 1978.

[9] Roberts, J.W. and Virtamo, J. (1991) "The Superposition of Periodic Cell Arrival Streams in an ATM Multiplexer", IEEE Trans. on Comm., Vol. 39, No. 2, Feb. 1991.

[10] Roberts, J.W., Mocci, U. and Virtamo, J. Eds. (1996) "Broadband Network Teletraffic" - Final Report of Action COST 242. Springer Verlag 